\title{
La réflexivité : exercice pédagogique et outil d'accompagnement aux cycles supérieurs
}

Andréanne Gélinas Proulx, Anne-Sophie Ruest-Paquette, Lilia A. Simões Forte, Megan Cotnam-Kappel, Caroline Fallu et Lucie Bartosova

\section{OpenEdition}

Édition électronique

URL : http://journals.openedition.org/ripes/672

DOI : 10.4000/ripes.672

ISSN : 2076-8427

Éditeur

Association internationale de pédagogie universitaire

Référence électronique

Andréanne Gélinas Proulx, Anne-Sophie Ruest-Paquette, Lilia A. Simões Forte, Megan Cotnam-Kappel, Caroline Fallu et Lucie Bartosova, "La réflexivité : exercice pédagogique et outil d'accompagnement aux cycles supérieurs », Revue internationale de pédagogie de l'enseignement supérieur [En ligne], 28(2) I 2012, mis en ligne le 06 novembre 2012, consulté le 07 septembre 2020. URL : http:// journals.openedition.org/ripes/672; DOI : https://doi.org/10.4000/ripes.672

Ce document a été généré automatiquement le 7 septembre 2020

Article L.111-1 du Code de la propriété intellectuelle. 


\title{
La réflexivité : exercice pédagogique et outil d'accompagnement aux cycles supérieurs
}

\author{
Andréanne Gélinas Proulx, Anne-Sophie Ruest-Paquette, Lilia A. \\ Simões Forte, Megan Cotnam-Kappel, Caroline Fallu et Lucie Bartosova
}

\section{Introduction}

1 La réflexivité constitue un processus cognitif qui « est devenu autant une exigence scientifique qu'une condition anthropologique " en sciences sociales (Gaucher, 2009, p. 8). C'est ce que nous avons constaté, en tant qu'étudiantes, lorsque notre professeure nous a demandé de rédiger un texte réflexif, qui a fait l'objet d'une évaluation formative et certificative, découlant d'un projet de recherche qualitative mené dans le cadre d'un cours intitulé « Recherche qualitative II » aux cycles supérieurs, c'est-à-dire aux $2^{\mathrm{e}}$ et $3^{\mathrm{e}}$ cycles universitaires. Cette expérience réflexive, engendrée par une immersion dans le processus de recherche qualitative et portant sur notre cheminement de chercheure depuis le début du cours, a suscité maintes remises en question et prises de conscience, de telle sorte que nous voulions en discuter à la suite $\mathrm{du}$ cours. Au terme d'une rencontre, nous avons décidé d'analyser nos textes autoréflexifs pour participer au dialogue concernant la réflexivité aux cycles supérieurs. Cette étude s'inscrit donc dans le courant des études qualitatives exploratoires de type co-construction auto-ethnographique (Cord \& Clements, 2010 ; Ellis, 2007), ce qui la distingue d'autres recherches menées sur la réflexivité. C'est entre les mois de mai et octobre 2010 que nous avons effectué la co-analyse de six textes autoréflexifs produits individuellement. Ce dit exercice réflexif a largement influencé notre développement en tant que personnes humaines, étudiantes et apprentieschercheures, d'où l'importance de partager nos données réflexives dans cet article. La question qui sous-tend cet article est la suivante: sur quels aspects de notre cheminement personnel, de notre parcours à titre d'étudiantes et de notre progression en tant que chercheures d'une étude qualitative sommes-nous portées à réfléchir dans 
le cadre d'un exercice de réflexivité ? Nos résultats font valoir en quoi la réflexivité est bénéfique tant pour la communauté scientifique qui s'intéresse à la pédagogie universitaire que pour les professeurs et étudiants aux cycles supérieurs.

Le présent article est organisé comme suit. Nous commencerons par un survol des écrits portant sur la pratique réflexive, l'écriture réflexive et les aspects théoriques liés au journal de bord. Nous développerons ensuite nos choix méthodologiques avant de présenter nos résultats. La discussion porte sur la pertinence de la réflexivité comme exercice pédagogique et comme outil d'accompagnement pour des étudiants aux cycles supérieurs.

\section{La pratique réflexive}

Plusieurs chercheurs et praticiens se sont penchés sur le thème de la réflexivité dans la recherche et la pratique, en éducation comme ailleurs, depuis le début du $\mathrm{XX}^{\mathrm{e}}$ siècle. Le terme revêt un caractère polysémique, façonné au gré des expériences et de l'entendement de ces professionnels, dont le premier fut Dewey en 1933 (Ruth-Sahd, 2003).

4 Selon Dewey, la pratique réflexive est une activité intellectuelle qui découle de la raison et s'apparente à la pensée critique à des fins de prudence et de prévoyance dans le respect d'une conduite scientifique et professionnelle, notamment afin de pallier les effets de l'instinctivité et de l'impulsivité (cité dans Fendler, 2003 ; Ruth-Sahd, 2003). À la manière de Dewey, d'autres auteurs entrevoient la réflexivité comme un instrument de professionnalisation, mais, à l'inverse de leur prédécesseur, la réflexivité qu'ils promeuvent est motivée par l'intuition plutôt que par le désir de se conformer à une méthode scientifique (Fendler, 2003 ; Schön, 1994). Ce faisant, Schön (1994) fait valoir le savoir-faire qui est contenu dans l'action des professionnels et qui se matérialise au fil de leurs réflexions. Il dénonce ainsi le modèle de la science appliquée « qui nous amène à penser que la pratique intelligente est une application du savoir théorique destinée à résoudre des problèmes » (p. 77).

Une telle réflexion peut être subdivisée en trois catégories : la réflexion dans l'action, sur l'actionet pour l'action (Schön, 1990, cité dans Ruth-Sahd, 2003 ; Schön, 1994). Dans le premier cas, le professionnel réfléchit spontanément lorsque l'action se déroule, puisant intuitivement dans sa conscience, ses connaissances et sa pensée critique afin de contrôler ou de modifier ses actions en cours (Deum, 2004 ; Perrenoud, 2004 ; RuthSahd, 2003 ; Schön, 1994). Dans le deuxième cas et selon les mêmes auteurs, la réflexion est intentionnelle et a lieu une fois l'action achevée dans le but de prendre un recul pour mieux décrire, comprendre, évaluer, puis expliquer les activités accomplies et en retirer des apprentissages ou des nouveaux savoirs (réflexion rétrospective), le tout pour mieux planifier, anticiper ainsi que se préparer à l'éventualité de situations et d'actions analogues (réflexion prospective). Pour Perrenoud (2004), elle recèle donc un caractère de continuité, car elle constitue une continuation de la réflexion amorcée dans l'action. Bien que la réflexion sur l'action se penche sur les actions accomplies, il ajoute qu'elle se réalise tantôt en cours d'action et tantôt dans l'après-coup. Par-delà ses fonctions méthodologiques, Perrenoud mentionne qu'elle permet au professionnel de comprendre ce que Bourdieu appelle l'habitus, c'est-à-dire « les biais et les limites de [sa] perception et de [sa] pensée dans l'action » (p. 41), ses raisonnements et réactions ainsi que « ses préjugés, ses goûts et ses dégoûts, ses peurs et ses désirs, ses obsessions 
aussi bien que ses cécités. Et parfois à s'intéresser à son inconscient [...]» (p. 43). Toutefois et au-delà de l'introspection de type nombriliste, comme le précise Bibauw (2010), ce type de réflexion « doit se fonder sur la confrontation à l'altérité, soit par l'échange verbal avec l'autre, soit par la mise en confrontation d'un état de savoir avec une nouvelle réalité »(p. 18). Les représentations intuitives qui émergent de la réflexion peuvent conséquemment être transformées, selon Perrenoud, en données d'analyse pouvant être appréhendées selon des cadres théoriques ou conceptuels personnels, collectifs (professionnel) ou "savants» (scientifique) (p. 44-45). Pour ce dernier auteur, il s'ensuit que la réflexivité renvoie à trois pratiques complémentaires : langagière, dialogique et sociale. La réflexion est langagière, car elle sollicite le langage et les concepts, et dialogique, puisqu'elle nous incite à anticiper un dialogue et des réactions. La dimension sociale apparaît dès que d'autres personnes sont impliquées dans la réflexion et lorsqu'elle est faite sur, avec ou bien contre les autres. Cette dernière dimension renvoie au concept de réflexion collective (ex. discuter des écrits, comparer les perceptions, apprendre dans la diversité et participer activement) (Deum, 2004). Enfin, la réflexion pour l'action détermine la finalité de la réflexion dans et sur l'action : réfléchir pour mieux orienter les actions à venir (Ruth-Sahd, 2003).

6 En milieu de travail et à la différence de la réflexion spontanée, la réflexivité « fait adopter une position d'extériorité ou une mise à distance qui facilite la construction d'un objet [...] et la (dé)construction du sens de l'action " (Legault, 2000, cité dans Donnay, 2001, p. 53), contribuant conséquemment au développement professionnel du praticien ou du chercheur. Puisqu'elle se dégage des apprentissages tirés et renégociés au fil des expériences professionnelles et en rapport au contexte, elle contribue au façonnement des capacités adaptatives (Bénaïoun-Ramirez, 2009) et assure la médiation entre l'image professionnelle de soi et celle de l'idéal professionnel (Jacquet-Mias, 1998, cité dans Bénaïoun-Ramirez, 2009). C'est ainsi qu'elle contribue à l'élaboration d'identités professionnelles qui sont elles-mêmes réflexives en raison des processus d'identisation (ou de différenciation) et d'identification (ou d'appartenance) qui les caractérisent (Tap, 1980, Tap et al., 1997, cités dans Bénaïoun-Ramirez, 2009).

7 En contexte de formation, la réflexivité peut autrement être qualifiée de "capacité métacognitive de retour de l'apprenant sur sa façon d'apprendre " (Romainville, 2007, cité dans Bibauw, 2010, p. 2). Bien que la réflexivité ne figure pas explicitement parmi les compétences visées à la maîtrise et au doctorat selon Prégent (2001), il y fait mention de pouvoir «mobiliser [...] une base de connaissances scientifiques", puis «faire preuve de rigueur, de sens critique, de probité et de respect de la propriété intellectuelle» (p. 2), lesquels sont compris dans une démarche réflexive. Nous en déduisons que le développement d'habiletés réflexives est constitutif de l'expérience étudiante aux $2^{\mathrm{e}}$ et $3^{\mathrm{e}}$ cycles. En outre, la réflexivité aux cycles supérieurs contribue à la (re)construction identitaire du chercheur en devenir puisqu'elle lui permet de saisir la raison d'être et les conséquences de ses actions avant, pendant et après son enquête sur le terrain (Cros, 2001). Ainsi, la posture de l'apprenant évolue de façon continue, lui permettant de découvrir sa propre voix de chercheur.

De la perspective de Perry (1970), les étudiants universitaires passent par des niveaux épistémologiques séquentiels de complexité cognitive croissante, notamment le dualisme, le relativisme et le compromis dans le relativisme. Autrement dit, ils évoluent d'un mode de pensée (ou vision du monde) souvent dichotomique ou absolutiste au relativisme qui admet que toute connaissance ou fait dépend d'une 
variété de variables. Ce modèle s'apparente à celui du jugement réflexif de Kitchener et King (1981) selon lequel le jugement des étudiants universitaires évolue d'un stade préréflexif à réflexif. Il est donc important d'adapter les pratiques pédagogiques selon le développement réflexif de l'étudiant.

\subsection{L'écriture réflexive}

9 L'écriture réflexive exige un effort de formulation qui nécessite une structuration et une clarification des pensées et conduites, puis permet «de mettre à distance, de transformer des représentations, de se relire et de prendre conscience de son propre développement, de se compléter» (Deum, 2004, p.6). Elle est donc à la fois transformative et formative. Précisons cependant que ce sont les pauses dans le rythme d'écriture qui aident à réfléchir sur sa pensée et ses actions, cette réflexion pouvant ensuite être consignée à l'écrit (Bibauw, 2010). Il s'ensuit que « l'écriture ne vient que dans un second temps, non comme exercice réflexif en tant que tel, mais comme effort d'intériorisation et de mise en évidence de ce qu'a enclenché la confrontation comme remise en question » (Bibauw, 2010, p. 17).

D'après Cros (2001), le chercheur trouve justement sa propre voie en négociant entre action et écriture, objectivité et subjectivité, pratique et théorie : «la voie est étroite mais elle conduit le praticien-chercheur, grâce à la recherche, à construire un espace 'transitionnel', espace symbolique entre lui et sa pratique de telle sorte à permettre sa propre émancipation " (p. 134). C'est le processus d'écriture qui fait valoir cet espace transitionnel, car le chercheur se distancie de sa recherche tout en poursuivant un double objectif : écrire pour communiquer et réfléchir. Bien que cette dualité crée des tensions, le chercheur évolue grâce aux convergences et divergences entre sa voix et sa voie de chercheur réflexif.

\subsection{Le journal de bord}

11 Le journal de bord (ou carnet de recherche) est l'un des instruments privilégiés dans une démarche d'écriture réflexive. Il constitue un «support d'autoformation qui a une portée à la fois rétroactive et proactive»(Deum, 2004, p. 8) ainsi qu'un outil d'autoévaluation et de développement d'une pensée autonome. En recherche, ce journal « est un support écrit ou informatique (ou les deux) dans lequel un [étudiant ou chercheur] consigne tout son travail intellectuel [y inclus ses réflexions] selon une structure thématique qui lui est propre " (Prégent, 2001, p. 44) à différents moments lors de la collecte, de l'analyse ou de l'interprétation des données (Charest, 1994 ; Prégent, 2001). Il inclut précisément des «traces écrites [...] dont le contenu concerne la narration d'événements [...] contextualisés " (Baribeau, 2005, p. 100), révélant des indices internes (idées, émotions, pensées, décisions) ou externes (faits, extraits de textes, descriptions d'une situation ou d'un lieu) pertinents à la recherche. De plus, ce journal illustre la position $\mathrm{du}$ chercheur, les changements encourus lors $\mathrm{du}$ déroulement de la recherche et ses interrogations concernant la collecte des données (Baribeau, 2005 ; Poisson, 1992). Il fonctionne alors comme un agent de liaison entre le processus de recherche et le développement du chercheur, lui permettant d'exposer les dilemmes encourus (Day, 2002). 
12 En somme, la tenue d'un tel journal facilite la rétention de certains événements et expériences, la création d'un lien entre les données et le chercheur qui les observe et les analyse, et la prise de distance par rapport à soi-même afin de s'examiner (Baribeau, 2005). Cette distanciation vis-à-vis de ses expériences permet au professionnel d'en retirer "des éléments généralisables et transmissibles", puis de partager son savoir d'expérience avec des collègues "afin de le réfléchir, de le discuter, voire de le retricoter dans l'intersubjectivité" (Deum, 2004, p.5). Il est d'ailleurs conseillé aux directeurs de mémoire ou de thèse d'encourager les étudiants sous leur tutelle à faire usage de ce dispositif, "car la tenue d'un tel carnet fait partie intégrante de la formation d'un chercheur", tout en présentant des avantages méthodiques et organisationnels (Prégent, 2001, p. 46).

\section{Méthodologie}

\subsection{Recherche qualitative exploratoire de type co-construction auto-ethnographique}

13 L'approche méthodologique privilégiée est celle d'une recherche qualitative exploratoire de type co-construction auto-ethnographique (Cord \& Clements, 2010 ; Ellis, 2007). Il est question du paradigme qualitatif, car nous adoptons une posture épistémologique constructiviste et un devis de recherche auto-ethnographique. Nos instruments de collecte et d'analyse de données ainsi que le caractère itératif et rétroactif de notre processus de recherche sont aussi typiques de cette tradition (Deslauriers \& Kérisit, 1997). En outre, le projet a évolué depuis son commencement en raison de nos nombreuses discussions. Cette transformation est caractéristique d'un mode méthodologique exploratoire dans lequel « la collecte des données et leur analyse devront s'ajuster à l'émergence de données inattendues et des intuitions analytiques » (Van der Maren, 2010, p. 75). Qui plus est, nous chevauchons les rôles d'étudiantes, de participantes et de chercheures. Bien que cette pluri-identité des six membres de la recherche soit originale, cette démarche s'apparente à l'auto-ethnographie, qui reflète l'expérience subjective d'un sujet-chercheur (Rondeau, 2011), ainsi qu'à la coconstruction auto-ethnographique, selon laquelle les participants et chercheurs sont les mêmes personnes (Cord \& Clements, 2010; Ellis, 2007). En dévoilant notre expérience réflexive, nous nous positionnons à l'encontre du discours académique traditionnel voulant que l'auteur s'efface (Ellis, 1997, citée dans Cord \& Clements, 2010). De plus, la méthode de la co-construction auto-ethnographique exige une étroite collaboration entre les sujets-chercheurs pendant l'étude (Cord \& Clements, 2010 ; Ellis, 2007). Nous avons conséquemment misé sur la coopération et la co-construction des savoirs durant notre recherche. Sur ce point, notre méthodologie est propice à l'institution d'une approche réflexive collective selon laquelle deux personnes ou plus s'engagent dans le processus réflexif pour co-créer un savoir (Cord \& Clements, 2010). Notre objectif en tant que chercheures est justement d'effectuer une co-analyse réflexive des textes réflexifs que nous avons rédigés comme étudiantes pour en dégager un savoir d'intérêt scientifique. L'auto-ethnographie préconise, entre autres, la réflexivité par l'écriture d'un journal de bord comme moyen pour comprendre l'expérience du(des) sujet(s)-chercheur(s) (Cord \& Clements, 2010 ; Rondeau, 2011). Nous verrons que l'écriture réflexive est au coeur de notre instrumentation. Sur le plan 
éthique, étant toutes impliquées dans les différentes phases de l'étude, nous n'avions pas à nous préoccuper d'obtenir des participants un consentement éclairé ni de révéler des informations privées (Ellis, 2007).

Enfin, l'unicité de notre démarche relève de notre volonté à nous approprier la parole en tant qu'étudiantes afin de promouvoir les savoirs ayant émergé de nos expériences, notamment à des fins pédagogiques, non seulement auprès de nos condisciples, mais également des professeurs et chercheurs qui nous encadrent et qui détiennent habituellement la légitimité d'énoncer les méthodes pédagogiques à privilégier. Ce faisant, nous renversons la relation de pouvoir en nous prononçant sur un sujet qui nous concerne, rappelant que «le chercheur universitaire n'a pas le monopole de la création de savoir, pas plus que le praticien celui du concret» (Donnay, 2001, p. 52). Notre étude répond alors aux recommandations de chercheurs qui suggèrent d'utiliser des devis et des méthodes alternatives pour accroitre les connaissances propres aux pratiques réflexives (Ruth-Sahd, 2003).

\section{2. Échantillonnage et posture}

Les données de notre étude découlent d'un exercice de réflexivité qui a été proposé à tous les étudiants inscrits à un cours doctoral de méthodologie de la recherche qualitative, c'est-à-dire à un total de six personnes. Nous sommes à la fois les six étudiantes de ce cours et les participantes de cette étude. Au moment de la collecte de données, nous étions toutes des femmes âgées entre 23 et 29 ans, dont quatre doctorantes et deux étudiantes à la maîtrise. Depuis ce temps, l'une des étudiantes à la maitrise a débuté son doctorat, alors qu'une autre a terminé sa maîtrise et a complété la formation à l'enseignement. Notre participation à l'étude était volontaire. Nous nous sommes attribuées des pseudonymes pour la présentation des résultats afin de protéger partiellement notre anonymat.

À la lumière des postures de création de savoir que propose Donnay (2001), nous nous positionnons à l'intersection entre une posture d'étudiant ${ }^{1}$ réflexif et de chercheuracteur. L'étudiant réflexif s'appuie sur ses savoirs d'expériences, parvient à décrire et rapporter sa démarche et s'efforce de comprendre ou d'extraire du savoir de ses actions et réflexions. Il est porté à effectuer un retour sur sa démarche, voire de la conceptualiser dans le but de diffuser oralement les savoirs créés, notamment par l'entremise du dialogue avec d'autres " pour partager, comparer, mettre en question, enrichir ses pratiques » (Donnay, 2001, p. 40). De son côté, le chercheur-acteur a pour finalité première de communiquer un savoir pratique encadré par des savoirs et des démarches scientifiques, ce savoir pouvant être compris par les étudiants, utilisé par les pédagogues en milieu universitaire, puis partagé avec la communauté scientifique. $C$ 'est à titre d'étudiantes réflexives et de chercheures-actrices que nous avons entamé cette étude et produit cet article.

\subsection{Instrumentation et collecte de données}

Nous avons privilégié un seul instrument de collecte de données, soit le texte autoréflexif. Ainsi, chaque participante a rédigé un texte de cinq pages correspondant à une version abrégée d'un travail plus élaboré d'environ 15 pages soumis lors de notre cours. Pour ce travail, la professeure nous incitait à "port[er] un regard sur [notre] 
cheminement à titre de chercheure depuis le début du cours». Elle a ensuite précisé que «la réflexion peut présenter les changements épistémologiques et/ou les insécurités épistémologiques. Elle peut aussi porter sur le rôle du chercheur et des participants dans la construction du savoir ». Nous pouvions aussi, dans nos textes réflexifs, insérer des extraits du journal de bord que nous avons tenu pendant notre projet de recherche respectif, ces extraits ayant été cumulés sur une période d'environ six semaines. Ces consignes s'inscrivent dans une démarche de réflexivité sur l'action (Deum, 2004 ; Perrenoud, 2004 ; Ruth-Sahd, 2003 ; Schön, 1994). Peu de balises ont été données pour nous guider dans ce travail. Cela a été bénéfique, car « la restriction des consignes de rédaction à une posture et à des contenus précis tend [...] à formater l'écriture réflexive et à la transformer en nouveau genre discursif à assimiler pour l'étudiant, plutôt qu'en outil d'autoapprentissage » (Bibauw, 2010, p. 18).

Par ailleurs, ce travail avait une valeur de $20 \%$ de la note finale. La professeure a recouru à une grille critériée détaillant les critères et niveaux de performance attendus pour réduire la subjectivité de l'évaluation (Berthiaume, David \& David, 2011), un A étant attribué lorsque «la réflexion est excellente. Elle identifie les éléments déclencheurs des apprentissages et des remises en question. Le cheminement effectué est rendu explicite». Bien que l'évaluation certificative "tend à faire oublier à l'apprenant l'enjeu formatif de l'exercice pour se préoccuper prioritairement de se conformer aux attentes de l'évaluateur" (Bibauw, 2010, p.17-18), les consignes d'écriture et d'évaluation étaient flexibles. Nous verrons d'ailleurs dans la présentation des résultats comment l'exercice de réflexivité fut formateur et déclencheur de partages indépendants des attentes de l'évaluatrice. En outre, ayant en main la grille critériée, nous avons pu auto-évaluer nos textes réflexifs avant de les remettre et mis en pratique nos capacités réflexives (Berthiaume, David \& David, 2011). Enfin, la professeure du cours a commenté par écrit nos textes réflexifs, mais ces commentaires n'ont pas été analysés pour cette recherche.

\subsection{Traitement et co-analyse des données}

19 Nous nous inspirons de l'analyse inductive générale, une méthode d'analyse de données proposée par Thomas (2006), puis reprise par Blais et Martineau (2006), "visant à 'donner un sens' à un corpus de données brutes mais complexes, dans le but de faire émerger des catégories favorisant la production de nouvelles connaissances en recherche» (p. 2). Elle comprend les étapes suivantes: «1. Préparer les données brutes; 2. Procéder à une lecture attentive et approfondie; 3. Procéder à l'identification et à la description des premières catégories ; 4 . Poursuivre la révision et le raffinement des catégories » (Blais \& Martineau, 2006, p. 6-8). Nous avons suivi les étapes prescrites, procédant à une co-analyse des données selon laquelle « le moment de l'analyse gagne à être partagé, ne fut-ce que pour la fiabilité du codage et la validation inter-juges progressive de la signification des données" (Mukamurera, Lacourse, \& Couturier, 2006, p. 123). Ainsi, trois d'entre nous ont codé deux textes réflexifs de façon indépendante afin de dégager les premières catégories et sous catégories. Ce codage en parallèle à l'aveugle favorise la rigueur de l'analyse (Blais \& Martineau, 2006). Une rencontre nous a permis de comparer notre codage et d'élaborer une liste préliminaire de catégories, la construction des catégories s'appuyant « sur une logique inductive : les catégories sont construites sur la base des interprétations des sujets » (Martinic, 2010, p. 198). Les trois mêmes chercheures ont ensuite lu et codé les 
six textes réflexifs en tenant compte de la liste des catégories préliminaires, puis ont noté les nouvelles catégories émergentes ainsi que les possibilités de fusion et de segmentation. Une seconde rencontre a permis aux analystes de repérer trois orientations réflexives: personnelle, scolaire et professionnelle. Elles y ont aussi délimité les catégories que nous allions utiliser pour la présentation des résultats selon leur récurrence dans l'ensemble des textes réflexifs. D'après Blais et Martineau (2006), «les chercheurs qui utilisent l'approche générale d'analyse inductive limitent habituellement le développement de leur cadre ou de leur modèle à la présentation et à la description des catégories les plus importantes ayant émergé de leur analyse » (p. 6). Grâce à une nouvelle liste épurée, nous avons pu procéder à un troisième codage des données avant d'amorcer l'analyse. La liste des catégories retenues est la suivante :

1. États affectifs :

- Anxiété, angoisse, panique, peurs, craintes, surprise, choc ;

- Niveau de confort, sécurité, confiance, doutes, hésitations, incompréhension ;

- Frustration, colère, révolte, haine ;

- Autres.

2. Étudiantes :

- Identité (hybride, différenciée) ;

- Cheminement (lectures, interactions et discussions avec nos collègues de classe/travail

d'équipe, travaux, processus).

3. Réflexions méthodologiques:

- Recherche qualitative ;

- Méthodes de collecte de données ;

- Méthodes d'analyse.

4. Réflexions épistémologiques :

- Posture ;

- Rôle dans la recherche ;

- Rapport aux participants.

5. Réflexion sur la réflexivité

Cette liste a été légèrement retravaillée pour la présentation des résultats.

21 Plutôt que de comparer leur codage afin d'en dégager un indice d'accord interjuge (Krippendorff, 2004), les trois analystes ont effectué l'analyse des données en s'appuyant sur tout le matériel réflexif codé, donc sur toutes les unités de textes repérées afin de tenir compte de notre interprétation subjective. Les analyses ont ensuite été vérifiées par les six sujets-chercheures pour assurer qu'elles respectaient leur point de vue. Notre positionnement endogène accroît d'ailleurs la justesse des résultats puisque nous pouvions en tout temps contre-vérifier et valider nos interprétations des textes réflexifs analysés augmentant ainsi la crédibilité de notre recherche (Pourtois \& Desmets, 2007).

\section{Présentation des résultats}

\section{Présentation des résultats}

Au terme du processus de codage, nous avons repéré trois catégories principales : la personne humaine, l'étudiante et l'apprentie-chercheure. Notre réflexivité a d'ailleurs porté sur cette hybridité identitaire: «ma personne apprentie-chercheure est aussi 
une personne hors campus et vice-versa » (Barbara); « non seulement je suis étudiante au doctorat, mais aussi jeune chercheure » (Gabriela) ; « je rédige ce rapport à la croisée des chemins entre ma personne humaine et l'académicienne que je deviens » (Océane). Nous choisissons alors de subdiviser la présentation des résultats en tenant compte de ce triptyque identitaire, mais reconnaissons que les frontières entre ces identités sont floues.

\subsection{Personne humaine ... et les états affectifs} qui engendrent le plus d'émotions, notamment de l'anxiété par peur de ne pas obtenir suffisamment de données, suivie par du plaisir sur le terrain (Julie), un sentiment de surprise en réaction aux imprévus (Barbara), de l'inconfort en tant qu'observatrice clandestine (Isabelle), de la frustration par la faute d'une absence de sujets à observer (Chantale), ainsi que de la tristesse et de l'impuissance vis-à-vis des comportements observés (Océane). Force est d'admettre que l'exercice d'observation est éprouvant pour la personne humaine qui transcende tout apprenti-chercheur. 
inductive [...] par peur de me tromper en utilisant une autre méthode, ou en forgeant la mienne propre».

Étant donné que le cours comportait une visée méthodologique de nature appliquée et qu'il se déroulait à raison de deux séances par semaine pendant six semaines, il n'était pas attendu que nous procédions à l'élaboration d'une problématique, ni à une revue des écrits pour procéder à la recherche de terrain. Selon les textes analysés, esquiver ces étapes de la recherche a chatouillé l'insécurité de deux étudiantes, lesquelles n'ont pu s'empêcher de recourir à des articles supplémentaires.

Pourtant, à la suite d'une première lecture des textes obligatoires portant sur l'épistémologie ou les méthodes de collecte et d'analyse de données, la moitié des étudiantes, y inclus Chantale, rapporte avoir vécu de l'incompréhension : "après la lecture d'un texte, l'incompréhension s'installe en moi, et il n'en ressort que l'impotence à comprendre les notions contenues dans le texte». Il y a, parmi ces mêmes étudiantes, deux d'entre elles qui signalent paradoxalement avoir vécu un rehaussement de leur sentiment de confiance par rapport aux méthodes à employer en raison de l'une des lectures obligatoires : « lire Feuerverger (2001) m'a ouvert les yeux et j'ai compris l'importance de m'écrire dans le journal de bord afin d'analyser les données en fonction de qui je suis comme chercheure » (Julie).

31 En somme, nous avons vécu des sentiments d'anxiété, de panique, de surprise, d'insécurité et d'incompréhension qui se sont manifestés en réaction à différents déclencheurs. Parmi eux, il y en avait qui se rattachaient au contenu du cours auquel nous étions inscrites. Cela étant dit, ces déclencheurs ont éveillé bien plus que des états affectifs. Examinons quels sont ces déclencheurs ainsi que les réflexions qu'ils ont provoquées au regard des étudiantes et apprenties-chercheures que nous sommes.

\section{2. Étudiante}

S'ensuit l'analyse des données réflexives qui relèvent plus explicitement de nos expériences comme étudiantes, soit l'apport des lectures et travaux ainsi que la nature de notre dynamique en tant que groupe-classe.

\subsubsection{Lectures et travaux}

Dans l'ensemble, les lectures, tout comme les travaux prescrits dans le cours, constituent, selon les données réflexives, des catalyseurs en regard des apprentissages méthodologiques acquis, des remises en question épistémologiques survenues ainsi que des prises de conscience générées : « les éléments suivants ont contribué à déclencher soit mon apprentissage soit mes remises en question: la lecture des articles [...] et finalement la réalisation des travaux » (Gabriela).

34 Julie compare d'ailleurs certains des chercheurs dont les articles apparaissaient au menu du cours à des « guides ». Si les ouvrages scientifiques correspondent à des guides et que les étudiantes sont appelées à poursuivre leur cheminement sans l'aide de ceuxci, il n'est pas étonnant qu'elles en éprouvent de l'insécurité, ce qui pourrait expliquer le malaise évoqué sous la rubrique «États affectifs ».

Quant aux travaux, nous avons, en tant qu'étudiantes, partagé mutuellement notre premier travail d'analyse ${ }^{2}$. Nous avons, de notre propre initiative, commenté quelques travaux et avons pu bénéficier de la rétroaction de nos pairs. Aux yeux des étudiantes, 
ce partage est porteur de prises de conscience importantes : "C'est d'ailleurs dans le contexte de cet échange que l'on m'a fait remarquer mon silence en regard du travail d'équipe. Sans m'en rendre compte, je refusais de m'intégrer à un groupe qui était prêt à m'accueillir » (Océane) ; «Lors de ma première lecture de leurs analyses, j'ai ressenti un certain niveau de panique puisqu'elles ne ressemblaient pas à la mienne. Je trouve ça intéressant que ma réaction initiale prétendait que différent était nécessairement mauvais [...] C'était une très belle expérience de développement parce que j'ai vu comment six chercheures peuvent toutes interpréter les mêmes données de façon différente, mais intéressante » (Isabelle).

Pour conclure, les étudiantes interagissaient avec les lectures, interprétant leur contenu pour mieux négocier leurs orientations méthodologiques et épistémologiques. Ces négociations ont ensuite été consignées sous forme de travaux, lesquels ont été évalués par notre professeure et commentés par nos pairs. Les étudiantes ont ainsi pu interagir avec la rétroaction fournie et donc renégocier les orientations précédemment délimitées. Sur ce point, la nature interactive et sociale de notre démarche a été mise en avant dans l'ensemble de nos textes réflexifs. La section suivante se réfère justement à ces réflexions.

\subsubsection{Dynamique en tant que groupe-classe}

Précisons d'abord que nous formions, en tant que groupe-classe et à la recommandation de notre professeure, une équipe de recherche. Nous avons conséquemment adopté un esprit de collaboration et d'ouverture favorable au respect des autres, au modelage et au maintien d'une atmosphère d'apprentissage sécuritaire : " [...] je n'ai pas senti la pression de devoir 'savoir' mais de savoir 'questionner' ce que je ne savais pas. Tout cela dans une atmosphère agréable et respectueuse. C'est comme cela que je me suis sentie moins intimidée et décidée à apprendre » (Barbara).

À la manière des lectures et travaux, nos interactions entre condisciples et avec notre professeure constituent, au regard des six étudiantes, le tremplin de multiples apprentissages, réflexions, clarifications ou remises en question d'ordre méthodologique, épistémologique ou identitaire : «Grâce à l'équipe de recherche, j'ai raffiné mon positionnement épistémologique [...]» (Julie) ; « [Nos] discussions ont par ailleurs formé la nature de mon projet de recherche, en ont modifié les étapes ou ont alimenté mes réflexions [...]» (Chantale).

Certaines parmi nous se questionnaient néanmoins à l'égard de leur rôle ou positionnement au sein du groupe-classe: «bien que nous formions une équipe de recherche, je ne parvenais pas à croire que j'en faisais partie » (Océane); «on est tellement un petit groupe que j'ai l'impression qu'on s'attend à ce que je contribue aux discussions » (Isabelle); «je crois que mon plus grand apport a été ma présence de chercheure jusqu'alors inexpérimentée : cela a permis à d'autres de réfléchir sur leurs pratiques afin de me les expliquer » (Chantale).

Tout compte fait, l'apprentissage, tout comme la construction de notre identité en tant qu'étudiante, est, en ce qui nous concerne, un processus qui relève notamment de coconstructions et de négociations sociales avec les écrits, nos pairs et la professeure du cours : «J'ai réalisé toute l'importance de s'entourer de collègues avec qui il est possible d'échanger des idées et de confronter nos conceptions. Je considère dorénavant que les partenaires sont vitaux pour mener une recherche rigoureuse. » (Julie). 
41 Outre la personne humaine et l'étudiante que nous sommes, nos réflexions portent également sur notre cheminement d'apprentie-chercheure. La prochaine section est vouée aux thèmes qui font l'objet de ces réflexions.

\subsection{Apprenties-chercheures}

À la lumière des textes autoréflexifs, il ressort que nous nous percevons aussi comme des apprenties-chercheures. Nous nous approprions cette identité à la suite des tâches de recherche accomplies dans le cours. Endossant cette posture, nous réfléchissons aux aspects méthodologiques et épistémologiques de la recherche. Enfin, nous nous engageons dans une réflexion sur la réflexivité du chercheur.

\subsubsection{Réflexivité sur la méthodologie}

Dans nos textes autoréflexifs, nous évoquons divers aspects méthodologiques de la recherche: nous nous positionnons envers la recherche qualitative et nous nous attardons sur les méthodes de collecte et d'analyse de données.

Pour Barbara, la recherche qualitative s'avère être « quelque chose de nouveau, comme un nouvel apprentissage constructif ». Quant à Chantale, elle "n'avai[t] aucune notion concrète de comment il fallait s'y prendre ». Par-delà le constat selon lequel elle apprend, Barbara anticipe la portée de ses apprentissages et forme une nouvelle attitude envers la recherche qualitative : «je vais commencer à regarder la recherche quantitative d'une manière différente. Je pense même que ces deux méthodologies [qualitative et quantitative] peuvent se compléter et nous amener à avoir une autre vision du monde ». Bref, bien que l'univers de la recherche qualitative relevait de l'inconnu pour trois apprenties-chercheures au début du cours, les constats à son égard s'avèrent positifs à la suite d'une période d'apprivoisement et de mûres réflexions.

Chacune de nous se penche également sur un minimum de deux méthodes de collecte de données parmi les quatre devant être utilisées lors de la recherche qualitative entreprise dans le cours. Par exemple, nous percevons les avantages et limites du récit de vie, proposons des façons de pallier ses lacunes et croyons pouvoir réinvestir ces découvertes dans de futures recherches : « [...] les récits de vie sont des représentations imparfaites et incomplètes de souvenirs remémorés. [...] il aurait fallu procéder à un retour sur les récits de vie ou à des collectes de données subséquentes auprès des auteures [...]» (Océane). Certaines étudiantes exposent les facteurs se rapportant à l'entrevue qui affectent la rigueur méthodologique (contamination des données). Nous réalisons aussi qu'il est ardu de maintenir notre position épistémologique lors de l'utilisation des outils de collecte de données : «Bien qu'avant d'effectuer l'entrevue, j'avais dans l'idée de co-construire le savoir avec la personne interviewée [...] je me suis rendue compte par la suite que je proposais des réponses dans mes questions et que je portais souvent un jugement dans celles-ci » (Chantale). La nature de nos réflexions sur l'observation est davantage affective, la conscience de ces états émotionnels nous permettant de nous questionner sur l'utilisation future de cette méthode: "L'observation clandestine est alors plus difficile à vivre qu'une observation participante et cette expérience a dévoilé que je préfère de loin la deuxième option » (Isabelle). Quoique le journal de bord suscite moins de réflexions, trois d'entre nous en avons relevé l'importance en recherche qualitative, dont Chantale: "Je m'aperçois qu'un journal de bord aurait été vital à la progression de la recherche et aux constantes 
remises en question [...]. Je constate donc que ce manque d'information a des répercussions sur ma qualité d'analyse et d'introspection de ma démarche [...]». En résumé, nous semblons avoir fait plusieurs constatations a posteriori concernant les méthodes de collecte de données, lesquelles pourront être réinvesties dans de futures recherches.

Nos réflexions portent aussi sur le processus d'analyse des données ainsi que sur les outils d'analyse. Leur nature est, d'une part, affective et, d'autre part, rétrospective. Julie soulève, entre autres, que «le processus de l'analyse inductive générale m'a permis d'expérimenter l'approche cyclique de la recherche qualitative ». Par ailleurs, la réflexivité permet de consolider nos nouvelles connaissances afin de les transférer à d'autres situations. En réfléchissant à un commentaire que la professeure lui a fait dans le cours, Isabelle démontre qu'elle a saisi la portée du commentaire et qu'elle en tiendra compte ultérieurement lorsqu'elle interprétera des données.

Notre réflexion porte de surcroît sur un outil d'analyse de données, soit un logiciel utilisé pendant nos projets individuels. De fait, trois étudiantes commentent leur expérience, dont Julie qui reconnaît l'utilité de l'outil : «le logiciel [...], lui, est d'une grande aide». Océane soutient inversement que le logiciel «[...] nuisait à ma compréhension des données ainsi qu'à l'organisation de mes codes ».

Somme toute, diverses questions et préoccupations méthodologiques sont abordées dans l'exercice de réflexivité des apprenties-chercheures. Il en va de même pour l'épistémologie.

\subsubsection{Réflexivité sur l'épistémologie}

Pour la plupart, nos textes réflexifs dévoilent notre posture épistémologique, notre rôle dans la recherche et le rapport entretenu avec les participants de la recherche.

Nous nous questionnons toutes sur notre posture épistémologique et parvenons presque toutes à en choisir une : «[...] j'ai réalisé que [l'épistémologie] qui me convient le plus dans ma recherche ou qui correspond à mon idéologie est le paradigme constructiviste " (Gabriela). Certaines choisissent le constructivisme en fonction des implications méthodologiques qui en découlent. Isabelle valorise notamment la «coconstruction » du savoir avec les participants. Pour Océane, c'est la « vision du monde » qu'apporte cette épistémologie qui l'influence. En rétrospective, certaines réalisent que leur posture et leur conduite se contredisent: «[...] j'ai tenté d'adopter le paradigme co-constructivisme, j'estime qu'il a toutefois été difficile de rester à l'intérieur des frontières de ce paradigme dû à diverses contraintes comme le temps, l'éthique et les objectifs du cours " (Julie). Pour terminer, une étudiante soutient qu'elle chevauchera deux paradigmes : «Je vais balancer entre une posture post-positiviste [...] et parfois constructiviste » (Barbara).

51 Cinq parmi nous réfléchissent aussi à nos rôles dans le processus de recherche. Deux reconnaissent qu'elles sont chercheures, mais aussi participantes. Julie, citant Gaucher, signale qu'elle a " un statut de l'entre-deux », tandis qu'Isabelle se dit « entre frontières quant à mon statut pour cette recherche». Par ailleurs, trois étudiantes avouent chevaucher les postures endogène et exogène lors de la collecte et de l'analyse des données. Par exemple, Barbara mentionne que «[...] je me considère encore 'outsider', dans mes méthodes d'analyse, mais dans certains cas dans l'interprétation des résultats [je me considère] comme 'insider'«. De son côté, Chantale se questionne quant aux 
implications futures de son rôle de chercheure: "Cela m'engagera-t-il à rester chercheure toute ma vie? ». Gabriela se questionne plutôt sur son rôle de chercheure au présent : «Quelle est ma responsabilité en tant que chercheure?». Ainsi, il semble que nous appréhendons le rôle du chercheur sous différents angles, montrant la diversité des préoccupations que nous avons lorsque nous nous immergeons dans la recherche.

De plus, les réflexions de quatre étudiantes dénotent l'importance qu'elles accordent à la voix des participants. Allant de pair avec l'épistémologie qu'elles privilégient, elles valorisent la co-construction du savoir ainsi que la voix des participants : «Quant aux narratrices, à l'interviewé et aux acteurs sociaux, leurs voix dans ce rapport, est primordiale " (Julie). Par ailleurs, deux étudiantes auraient préféré interagir différemment avec les participants lors de la collecte de données. Après des observations clandestines, Isabelle affirme qu'elle "[aurait] voulu interagir avec les gens dans le magasin » pour obtenir plus d'informations. Julie précise que « bien que je trouve intéressant et pertinent d'offrir aux participants de la recherche la chance de découvrir le chercheur, je ne peux pas dire que je suis allée aussi loin que [Feuerverger] dans cette démarche ". Nos propos témoignent de nos allées retours entre les modes rétrospectif et prospectif de la réflexivité.

L'analyse de nos réflexions sur le plan épistémologique nous permet de constater, dans une certaine mesure, nos apprentissages, voire notre évolution. De fait, la réflexivité comme activité de développement professionnel nous a permis de définir notre posture épistémologique, cerner (ou questionner) notre rôle en tant que chercheure et décrire le rapport (souhaité) aux participants de la recherche. Bien que certaines questions demeurent sans réponses, la réflexion épistémologique est néanmoins amorcée.

\subsubsection{Réflexion sur la réflexivité du chercheur}

54 Concernant la pratique réflexive en recherche, Isabelle précise ceci : «On m’a toujours avisée d'être une praticienne réflexive lorsque j'enseigne en salle de classe. [...] Ce même concept est aussi important pour une chercheure [...] ». D'autres étudiantes font également valoir les avantages de la réflexivité du chercheur. Il ressort que l'exercice de la réflexivité nous permet d'être autocritiques et de nous remettre en question dans la démarche de recherche à des fins de cheminement et de rigueur scientifique. Par exemple, Gabriela souligne que « j'ai rendu transparent mon processus intellectuel avec l'intention de mieux comprendre mon cheminement à titre de chercheure ». Nous reconnaissons aussi qu'une certaine forme de réflexivité, rendue disponible aux lecteurs de la recherche, permet d'accroître sa validité. À ce sujet, deux étudiantes, dont Julie, perçoivent la valeur du dévoilement de soi dans le rapport de recherche pour fournir aux lecteurs des informations pouvant expliquer leurs interprétations : " [se dévoiler] n'est pas considéré comme un biais ou des limites à la recherche, mais comme une façon de faire part de sa réalité pour mieux être compris [des lecteurs] ». Bien que nous reconnaissons toutes les bienfaits de la réflexivité, deux étudiantes, dont Barbara, l'associent à un défi, "car elle implique [...] une procédure cognitive, organisationnelle et émotionnelle différente de tous les travaux [...] produits jusqu'à présent ».

Bref, l'exercice de réflexivité du chercheur est soumis au même traitement réflexif que les expériences d'ordres affectif, scolaire, méthodologique et épistémologique mentionnées précédemment. 


\section{Discussion} qu'étudiantes à la maitrise ou au doctorat inscrites à un cours de recherche qualitative, employé l'écriture autoréflexive afin de consigner nos réflexions selon trois dimensions identitaires : les états affectifs de notre personne humaine; notre rapport aux lectures, aux travaux et à la dynamique de classe comme étudiante; puis notre rapport à la réflexivité ainsi qu'aux aspects méthodologiques et épistémologiques comme apprentie-chercheure. Dans sa grille d'évaluation d'une pratique réflexive, Deum (2004) identifie les mêmes dimensions : «je » personne ; «je » étudiant ; «je» professionnel (p. 11). Par ailleurs, nos résultats suggèrent que la réflexivité est bénéfique aux cycles supérieurs. Les prochaines sections en témoigneront.

\subsection{L'écriture réflexive : exprimer l'affectif pour en tirer des apprentissages}

Nos données suggèrent que la personne humaine est fréquemment interpellée lors du parcours universitaire d'apprenties-chercheures, ce qui témoigne du caractère intuitif et éprouvant de la formation. Cela s'est concrètement manifesté en réaction à divers déclencheurs et par l'expression écrite d'une multitude d'émotions. D'après Perrenoud (2004), inspiré de Bourdieu, la réflexivité permet justement de rendre compte de l'habitus du chercheur, lequel englobe ses biais et limites, de même que ses raisonnements, réactions et émotions. L'écriture réflexive dans un journal de bord est d'ailleurs propice à la documentation des émotions du chercheur (Baribeau, 2005).

En ce qui nous concerne, nommer et décrire nos émotions à l'écrit nous a permis d'en prendre conscience, pour ensuite en décortiquer l'origine et la portée dans le cadre de notre cheminement universitaire et professionnel à des fins d'apprentissage et de rigueur professionnelle. La réflexivité est ainsi favorable au développement de la métaémotion, laquelle comprend une conscience accrue de ses émotions (nature et causes) et des moyens de les gérer (Pons, Doudin, Harris, \& de Rosnay, 2002).

Ayant rédigé nos écrits réflexifs à la première personne, puis ayant fait preuve de transparence en partageant nos textes avec notre professeure, entre nous et sous la forme d'un article, nous avons exposé notre vulnérabilité, sachant très bien que nous mettions (et mettons) à nue nos forces et fragilités, puis que nous courions (et courons) le risque de subir le jugement critique d'autrui (Ellis, 2007 ; Krieger, 1991). Cependant, écrire "sa faiblesse ou sa force, c'est déjà la reconnaître et en faire une base de réflexion sur laquelle s'appuyer » (Deum, 2004, p. 6). La réflexivité se résume d'ailleurs à un «espace transitionnel» entre le chercheur et sa pratique à des fins d'émancipation (Cros, 2001).

Nous avons d'ailleurs transformé nos représentations affectives et intuitives en données d'analyse pouvant être appréhendées selon des cadres conceptuels personnels, collectifs et "savants» (Perrenoud, 2004, p. 44-45). Cet article est le fruit de cette transformation. Pour ce faire, nous avons adopté une "position d'extériorité ou une mise à distance » pour mieux déconstruire le sens de l'action, puis construire un objet d'étude (Legault, 2000, cité dans Donnay, 2001, p. 53). L'écriture réflexive, laquelle nécessite une prise de distance par rapport à soi-même, est favorable à une telle prise

Revue internationale de pédagogie de l'enseignement supérieur, 28(2) | 2012 
de conscience, à la gestion des émotions qui émergent et à la transformation du chercheur qui s'écrit (Deum, 2004).

61 Nos données concordent par ailleurs avec les écrits d'auteurs qui étudient le rôle de l'affectivité dans l'apprentissage (Côté, 2002 ; Lafortune, Mongeau, Daniel, \& Pallascio, 2002). À ce propos, il a été démontré qu'une démarche expérientielle, qui invoque les dimensions affective, cognitive et comportementale de la personne, améliore le rendement scolaire chez des élèves du primaire et des étudiants aux cycles supérieurs (Côté, 2002). Étant donné que l'écriture autoréflexive dans un carnet de recherche comprend la narration d'événements vécus selon des indices internes (idées, émotions, pensées, décisions) et externes (faits, extraits de textes, descriptions d'une situation ou d'un lieu) (Baribeau, 2005), nous avançons qu'elle s'inscrit dans une telle démarche expérientielle. Nous en déduisons que l'écriture réflexive telle que nous l'avons pratiquée a possiblement influé sur notre réussite scolaire. En plus, Lafortune et ses collègues (2002) montrent que l'acte de "philosopher " sur une matière anxiogène assure à l'élève un "meilleur contrôle sur les moyens d'apprentissage mis à [sa] disposition» (p. 30). Comme ce fut notre cas, l'expression écrite de nos émotions, suivie de discussions, nous a permis d'en désamorcer les effets pour mieux assurer un contrôle sur notre apprentissage.

\subsection{L'étudiant réflexif et la consolidation des apprentissages aux cycles supérieurs}

62 Nos résultats indiquent que l'écriture autoréflexive en tant qu'exercice pédagogique aux cycles supérieurs répond aux exigences de la formation à la recherche, dont les compétences visées incluent notamment «mobiliser [...] une base de connaissances scientifiques », puis " faire preuve de rigueur, de sens critique, de probité et de respect de la propriété intellectuelle " (Prégent, 2001, p. 2). Apprendre est une condition de la mise en pratique de telles compétences, tout comme ces compétences facilitent le processus d'apprentissage. Sur ce point, la réflexivité en tant que "capacité métacognitive " nous a permis, sous la forme d'écrits réflexifs, de verbaliser nos apprentissages, puis d'effectuer un retour sur la façon dont nous les avons réalisés (Romainville, 2007, cité dans Bibauw, 2010, p.2). Nous avons ainsi intériorisé les apprentissages réalisés et mis en pratique dans le cours, puis adapté notre démarche et nos attitudes à la lumière de nos réflexions. La réflexivité est d'ailleurs favorable au façonnement des capacités adaptatives des professionnels (Bénaïoun-Ramirez, 2009). Elle nous a également permis d'effectuer un retour sur nos savoir-faire, contribuant à leur consolidation (Schön, 1994).

63 Nous avons d'ailleurs profité de l'écriture réflexive pour interpréter le contenu des lectures du cours et négocier nos orientations méthodologiques et épistémologiques en interaction avec les matériaux proposés. La rétroaction fournie par la professeure et nos pairs lors de l'évaluation des travaux et des discussions de groupe, de même que les confrontations qui s'en dégageaient, nous poussaient ensuite à reformuler nos orientations, toujours sous la forme d'écriture réflexive. Cela renvoie au caractère langagier, dialogique et social inhérent à la réflexivité (Perrenoud, 2004) et à l'apport de la confrontation à l'altérité dans la poursuite d'une démarche réflexive (Bibauw, 2010; Deum, 2004), de même qu'à l'écriture réflexive en tant qu'effort "d'intériorisation et de mise en évidence » des remises en question qui ressortent de 
telles confrontations (Bibauw, 2010). Tel que nous l'avons expérimenté, une pratique réflexive collective permet aussi à l'étudiant de "prendre une part active dans sa formation »(Deum, 2004, p. 17).

Puisque l'exercice de réflexivité auquel nous nous sommes adonnées a eu lieu dans l'action et sur l'action (en rétrospective, après la collecte et l'analyse des données, puis après le cours auquel nous étions inscrites), nous avons pu mieux décrire, comprendre, évaluer et expliquer nos actions (Deum, 2004 ; Perrenoud, 2004; Ruth-Sahd, 2003 ; Schön, 1994), celles-ci étant éclairées par nos apprentissages. Nous sommes ainsi de l'avis que la réflexivité chez l'apprenti-chercheur permet de clarifier les étapes d'une recherche, puis d'orienter ses choix méthodologiques et épistémologiques, et ce, au rythme de ses interactions, prises de conscience et remises en question ainsi que dans le respect de ses intérêts et limites, d'autant plus quand il recourt au journal de bord. Ce journal est justement reconnu comme outil propice à l'autoévaluation (choix, biais, conduite) et au développement d'une pensée autonome (Deum, 2004). Tel Prégent (2001), nous conseillons donc aux mentors (professeurs ou directeur de mémoire ou de thèse) d'encourager leurs étudiants à faire usage d'un tel dispositif.

\subsection{L'étudiant réflexif : construire son identité professionnelle}

Ayant consigné notre démarche de recherche et les discussions de groupe dans un journal de bord, puis ayant eu recours à ce journal pour rédiger notre texte autoréflexif, nous avons pu remarquer notre cheminement. Le journal de bord fonctionne justement comme un agent de liaison entre le processus de recherche et le développement personnel du chercheur (Day, 2002). Reconnaître ce développement, tout comme les remises en question et prises de conscience qui l'ont façonné, est bénéfique au modelage d'une identité professionnelle de chercheur (Bénaïoun-Ramirez, 2009 ; Cros, 2001). Cette reconnaissance permet aussi au chercheur de tirer profit des apprentissages rétrospectifs en vue de prévoir ses orientations futures (Deum, 2004 ; Perrenoud, 2004 ; Ruth-Sahd, 2003 ; Schön, 1994).

Toute réflexion écrite ou partagée à l'oral portant sur la méthodologie et l'épistémologie s'est avérée utile dans la construction de notre identité en tant que chercheure, dans le raffinement de notre posture de recherche et dans le développement de notre habileté à décrire et justifier nos choix. Nous avons toutes, dans une certaine mesure, évolué d'un mode de pensée plus ou moins dichotomique et absolutiste (ex. recherche qualitative versus quantitative, travail individuel versus travail en équipe) vers un mode de pensée plus relativiste (Perry, 1970). Quelques-unes n'avaient d'ailleurs jamais effectué, en toute conscience, un exercice réflexif. Nous pourrions donc avancer qu'elles sont passées d'un stade préréflexif à réflexif (Kitchener \& King, 1981), ce qui constitue une transition notoire dans le parcours des apprentischercheurs.

La pratique réflexive nous a aussi permis de nous positionner au sein de l'équipe de recherche que nous formions dans le cours, puis de remettre en question ce positionnement, favorisant un rapport plus équilibré et équitable. Concernant la recherche réalisée dans le cours, nos écrits réflexifs nous ont également permis de nous positionner en relation aux participants et à l'objet d'étude, si bien que nous avons pu identifier nos dilemmes et contradictions, puis clarifier notre rôle dans la recherche. Le journal de bord illustre notamment la position du chercheur et ses interrogations vis-à- 
vis de la collecte des données (Baribeau, 2005 ; Poisson, 1992), tout en permettant d'exposer les dilemmes encourus (Day, 2002).

En outre, nous avons, dans notre cours, partagé mutuellement et commenté notre premier travail d'analyse, sans compter que nous avons lu nos textes autoréflexifs pour la rédaction de cet article. Ces échanges concernant le contenu de nos écrits ont favorisé une démarche réflexive fondée sur la collaboration et la confrontation à l'altérité, soit deux conditions tributaires d'une pratique réflexive réussie (Bibauw, 2010 ; Deum, 2004 ; Perrenoud, 2004). Nous avons ainsi constaté que nous vivions toutes des insécurités, préoccupations et remises en question comparables. Cela nous a incitées à relativiser ce que nous éprouvions individuellement. Selon nos expériences, ces états affectifs aux cycles supérieurs sont habituellement vécus dans l'isolement, ce pour quoi le partage et la normalisation de ces états ont été réconfortants dans notre cheminement universitaire.

De plus, cette transparence entre condisciples permet de nuancer l'image de l'idéal professionnel et de recadrer son image professionnelle de soi (Jacquet-Mias, 1998, cité dans Bénaïoun-Ramirez, 2009). Nous sommes d'ailleurs parvenues, au cours de notre démarche réflexive collaborative, à surpasser certaines insécurités. Notre confiance en soi a été bonifiée, ce qui constitue un indice de transformation identitaire, la confiance étant l'un des trois sentiments principaux de l'identité (Fortier, 1997). C'est donc par l'intermédiaire d'un processus d'identification, mais aussi d'identisation, que notre identité professionnelle, elle-même réflexive, a pu évoluer (Tap, 1980, Tap et al., 1997, cités dans Bénaïoun-Ramirez, 2009).

Bref, notre (re)construction identitaire en tant qu'apprenties-chercheures, tout comme la consolidation de nos apprentissages, relève principalement d'interactions, de coconstructions et de négociations sociales, dont le médiateur principal fut l'écriture réflexive, la réflexivité étant en soit un instrument de professionnalisation (Fendler, 2003 ; Schön, 1994). Nous stipulons qu'il importe d'y recourir dans la formation de futurs chercheurs, l'écriture réflexive étant transformative et formative (Deum, 2004).

\subsection{La réflexivité : un outil d'accompagnement à promouvoir}

71 Nos résultats montrent la pertinence de la réflexivité comme exercice pédagogique, mais aussi comme outil d'accompagnement des étudiants aux cycles supérieurs. Nous postulons que la réflexivité, lorsqu'elle est partagée ou exercée conjointement entre l'étudiant et ses mentors, permet à ces derniers de saisir la nature des réflexions de l'étudiant, de sorte qu'ils puissent plus aisément l'encadrer, le guider, le soutenir et comprendre son cheminement. Elle leur permet aussi de mieux cerner la profondeur des réflexions de l'étudiant en question, de même que l'étendue de ses habiletés en matière d'analyse, le tout dans le but de mieux l'orienter afin qu'il puisse développer son plein potentiel comme chercheur.

72 Tel qu'élucidé plus tôt, le partage de nos travaux a contribué à l'émergence d'un dialogue authentique avec soi-même, entre nous, mais aussi entre notre professeure et nous. En ce sens, la réflexivité est un catalyseur de rétroaction (Bibauw, 2010), facilitant le dialogue entre l'étudiant et les ressources humaines à sa disposition. Nous proposons donc d'élargir l'« espace transitionnel » que constitue la réflexivité entre l'apprentichercheur et sa pratique (Cros, 2001) pour faire intervenir ses pairs et ses mentors. Rappelons que la réflexivité est à la fois langagière, dialogique et sociale (Perrenoud, 
2004). En tant que dynamique d'interaction, la réflexivité partagée provoque des conflits sociocognitifs qui peuvent être débattus et parfois résolus réflexivement, suscitant des changements de perspective et accroissant l'efficacité de l'accompagnement de l'étudiant (Lafortune \& Deaudelin, 2001; Lafortune, 2008). Cela renvoie une fois de plus à l'apport de la collaboration et de l'altérité dans une démarche réflexive réussie (Bibauw, 2010 ; Deum, 2004 ; Perrenoud, 2004).

Qui plus est, l'intervention d'un interlocuteur (ou le contact social) contribue au développement supérieur du fonctionnement cognitif (Vygotsky, cité dans Daniels, 2008). Lorsque l'étudiant apprend à anticiper l'intervention de ses mentors parce que ces derniers ont commenté ses réflexions et qu'ils ont extériorisé leurs propres réflexions, il peut alors plus aisément anticiper l'intervention d'autres interlocuteurs, y inclus celle de ses pairs, d'autres professeurs et des auteurs ou chercheurs étudiés.

Enfin, nous avons, par l'entremise de la réflexivité, ciblé nos difficultés pour mieux les surmonter ou pour concevoir, en négociations avec soi-même, avec nos pairs ou avec notre mentor, des alternatives viables. Par ailleurs, lorsque révélée au corps professoral, la réflexivité de l'étudiant permettrait d'identifier ses besoins et pourrait ouvrir la voie à de nouvelles initiatives pédagogiques (ateliers de formation spécialisés).

\subsection{Limites méthodologiques}

Malgré la richesse des résultats, il importe de souligner les limites de notre étude.

Premièrement, un logiciel informatique n'a pas été utilisé pour l'analyse des données. Quoique son utilisation faciliterait et accélérerait le codage dans le cas d'une étude aux données nombreuses (Lewis, 2004, Savoie-Zajc, 2000, cités dans Blais \& Martineau, 2006), il n'est pas exclu, comme nous avions un nombre restreint de documents à analyser, d'utiliser «[...] une grille d'analyse fabriquée 'à la main' pour faciliter le codage des données » (Blais \& Martineau, 2006, p. 8).

Deuxièmement, la triangulation des méthodes de collecte de données n'a pas été effectuée. Toutefois, les auteures de l'article, étant elles-mêmes les participantes de la recherche, ont eu plusieurs occasions pour commenter la co-analyse des données et ainsi compléter l'information lacunaire. Blais et Martineau (2006) signalent d'ailleurs que la vérification auprès des participants augmente la crédibilité de l'étude.

Troisièmement, les trois analystes ont codé différemment : l'une a codé de manière plus générale, s'attardant plutôt au sens des phrases ou des expressions qu'à chaque mot, alors que les deux autres ont codé d'une façon plus précise. L'expérience limitée des chercheures peut expliquer cette variation.

Enfin, le nombre restreint de participantes ainsi que la similitude de leur profil réduit la portée transférable de nos résultats. Il en va de même pour la réalisation d'un exercice réflexif dans le cadre d'un même cours. Il serait donc intéressant d'effectuer une recherche semblable avec un bassin plus nombreux et diversifié de participantschercheurs.

Malgré ces limites, nous estimons que les résultats présentés fournissent un éclairage unique sur la pratique réflexive aux cycles supérieurs. 


\section{Conclusion}

Tout compte fait, nous postulons que les professeurs aux cycles supérieurs auraient intérêt à favoriser une pratique réflexive collaborative et ouverte à l'expression des états affectifs de leurs étudiants, cette pratique étant fondée principalement sur l'écriture réflexive et le partage réflexif entre étudiants et mentors, puis entre condisciples.

En définitive, l'analyse de textes réflexifs de six étudiantes montre que la réflexivité est bénéfique aux cycles supérieurs pour les raisons suivantes: 1) il est souhaitable d'exprimer l'affectifsous forme d'écriture réflexive pour en tirer des apprentissages ; 2) l'étudiant réflexif est bien outillé pour consolider ses apprentissages ; 3) un tel étudiant construit une identité professionnelle viable; et 4) la réflexivité est une méthode d'accompagnement de l'étudiant. L'écriture réflexive est d'ailleurs propice au cheminement personnel, scolaire et professionnel de l'apprenti-chercheur, tant du point de vue de la méta-émotion, de ses compétences, que de son identité.

Bien que d'autres chercheurs aient pu avancer des constats comparables, il était important pour nous, en tant qu'étudiantes, de forger nos propres conclusions et de les exprimer publiquement afin de participer au dialogue en matière de réflexivité aux cycles supérieurs. Nous conseillons d'ailleurs à tout apprenti-chercheur d'entamer un tel exercice afin de faire valoir ses savoirs d'expérience tout en poursuivant sa démarche réflexive.

\section{BIBLIOGRAPHIE}

Baribeau, C. (2005). Le journal de bord de chercheur. Recherches qualitatives, Hors Série(2), 98-114.

Bénaïoun-Ramirez, N. (2009). Identités professionnelles entre réflexivité et adaptation : histoires de vie professionnelle de maîtres-formateurs. Recherche et formation, 60, 135-150.

Berthiaume, D., David, J., \& David, T. (2011). Réduire la subjectivité lors de l'évaluation des apprentissages à l'aide d'une grille critériée : repères théoriques et applications à un enseignement interdisciplinaire. Revue internationale de pédagogie de l'enseignement supérieur, 27(2). Repéré à http://ripes.revues.org/524

Bibauw, S. (2010). Écriture réflexive et réflexion critique dans l'exercice du compte rendu. Revue internationale de pédagogie de l'enseignement supérieur, 26(1). Repéré à http://ripes.revues.org/358

Blais, M., \& Martineau, S. (2006). L'analyse inductive générale : description d'une démarche visant à donner un sens à des données brutes. Recherches qualitatives, 26(2), 1-18.

Charest, P. (1994). Ethnométhodologie et recherche en éducation. Revue des sciences de l'éducation, 20(4), 741-756.

Cord, B., \& Clements, M. (2010). Reward through collective reflection : An autoethnography. $e$ Journal of Business Education \& Scholarship of Teaching, 4(1), 11-18. 
Côté, R. L. (2002). Faire des émotions et de l'affectivité des alliés dans le processus d'enseignement-apprentissage. Dans P. Mongeau \& L. Lafortune (dir.), L'affectivité dans l'apprentissage (p. 85-114). Sainte-Foy, QC : Presses de l'Université du Québec.

Cros, F. (2001). La recherche professionnelle : épistémologie et écriture. Dans M. Mackiewicz (dir.), Praticien et chercheur : parcours dans le champ social (p. 119-134). Paris : L'Harmattan.

Daniels, H. (2008). Vygotsky and research. New York : Routledge.

Day, E. (2002). Me, my*self and I : Personal and professional re-constructions in ethnographic research. Forum : Qualitative Social Research, 3(3). Repéré à http://www.qualitative-research.net/ index.php/fqs/article/view/824/1790

Deslauriers, J. P., \& Kérisit, M. (1997). Le devis de recherche qualitative. Dans J. Poupart, J.-P. Deslauriers, L.-H. Groulx, A. Laperrière, R. Mayer, \& A. P. Pires (dir.), La recherche qualitative : enjeux épistémologiques et méthodologiques (p. 85-112). Montréal, QC : Gaëtan Morin Éditeur.

Deum, M. (2004, août). Développer la pratique réflexive et amorcer la professionnalisation en formation initiale des enseignants par la rédaction du journal de bord : analyse d'un dispositif belge de formation au préscolaire. Communication présentée au $9^{\mathrm{e}}$ colloque de l'AIRDF, Québec, Québec.

Donnay, J. (2001). Chercheur, praticien même terrain ? Recherches qualitatives, 22, 34-53.

Ellis, C. (2007). Telling secrets, revealing lives relational ethics in research with intimate others. Qualitative Inquiry, 13(1), 3-29.

Fendler, L. (2003). Teacher reflection in a hall of mirrors : Historical influences and political reverberations. Educational Researcher, 32, 16-25.

Fortier, C. (1997). Les individus au cœur du social. Québec et Ottawa : Les presses de l'Université Laval.

Gaucher, C. (2009). De passeur de mots à médiateur de sens : affronter les risques méthodologiques d'une interprétation anthropologique de la quête identitaire des Sourds. Recherches qualitatives, 28(3), 6-18.

Kitchener, K., \& King, P. (1981). Reflective judgement : Concepts of justification and their relationship to age and education. Journal of Applied Developmental Psychology, 2, 89-116.

Krieger, S. (1991). Social Science \& the Self: Personal Essays on an Art Form. New Brunswick, NJ : Rutgers University Press.

Krippendorff, K. (2004). Content analysis : An introduction to its methodology. Thousand Oaks, CA : Sage.

Lafortune, L. (2008). Un modèle d'accompagnement professionnel et des compétences professionnelles pour l'accompagnement d'un changement. Sainte-Foy, QC : Presses de l'Université du Québec.

Lafortune, L., \& Deaudelin, C. (2001). Accompagnement socioconstructiviste : pour s'approprier une réforme en éducation. Sainte-Foy, QC : Presses de l’Université du Québec.

Lafortune, L., Mongeau, P., Daniel, M.-F., \& Pallascio, R. (2002). Philosopher sur les mathématiques : évolution du concept de soi et des croyances attributionnelles de contrôle. Dans L. Lafortune \& P. Mongeau (dir.), L'affectivité dans l'apprentissage (p. 29-50). Sainte-Foy, QC : Presses de l'Université du Québec.

Martinic, S. (2010). L'analyse structurale de contenu : quelle construction de catégories ? Dans L. Paquay, M. Crahay, \& J. M. De Ketele (dir.), L'analyse qualitative en éducation : des pratiques de recherche aux critères de qualité (p. 197-201). Bruxelles : De Boeck. 
Mukamurera, J., Lacourse, F., \& Couturier, Y. (2006). Des avancées en analyse qualitative : pour une transparence et une systématisation des pratiques. Recherches qualitatives, 26(1), 110-138.

Perrenoud, P. (2004). Adosser la pratique réflexive aux sciences sociales, condition de la professionnalisation. Éducation Permanente, 160, 35-60.

Perry, W. G. (1970). Forms of intellectual and ethical development in the college years : A scheme. New York, NY : Holt, Rinehart, and Winston.

Poisson, Y. (1992). La recherche qualitative en éducation. Québec, QC : Presses de l'Université de Québec.

Pons, F., Doudin, P.-A., Harris, P. L., \& de Rosnay, M. (2002). Métaémotion et intégration scolaire. Dans L. Lafortune \& P. Mongeau (dir.), L'affectivité dans l'apprentissage (p. 29-50). Sainte-Foy, QC : Presses de l'Université du Québec.

Pourtois, J.-P., \& Desmet, H. (2007). Épistémologie et instrumentation en sciences humaines (3éd.). Wavre : Éditions Mardaga.

Prégent, R. (2001). Encadrement des travaux de mémoire et de thèse : conseils pédagogiques aux directeurs de recherche. Montréal, QC : Presses internationales Polytechnique.

Rondeau, K. (2011). L'autoethnographie : une quête de sens réflexive et conscientisée au cœur de la construction identitaire. Recherches qualitatives, 30(2), 48-70.

Ruth-Sahd, L. (2003). Reflective practice : A critical analysis of data-based studies and implications for nursing education. Journal of Nursing Education, 42(11), 488-497.

Schön, D. A. (1994). Le praticien réflexif : à la recherche du savoir caché dans l'agir professionnel (traduit par D. Gagnon \& J. Heynemand). Montréal, QC : Les Éditions Logiques.

Thomas, D. (2006). A general inductive approach for analyzing qualitative evaluation data. American Journal of Evaluation, 27(2), 237-246.

Van der Maren, J.-M. (2010). Les recherches qualitatives : des critères variés de qualité en fonction des types de recherche. Dans L. Paquay, M. Crahay, \& J. M. De Ketele (dir.), L'analyse qualitative en éducation : des pratiques de recherche aux critères de qualité (p. 69-84). Bruxelles : De Boeck.

\section{NOTES}

1. Nous remplaçons " praticien » par « étudiant».

2. Spécifions qu'il s'agit d'un travail d'analyse à remettre pour notre cours et non de l'analyse réalisée pour cette étude.

\section{RÉSUMÉS}

Les résultats présentés dans cet article découlent d'une étude qualitative exploratoire de type coconstruction auto-ethnographique portant sur la réflexivité d'étudiantes aux cycles supérieurs, démarche qui exige que l'on porte un regard analytique et critique sur soi ainsi que sur sa 
position, son rôle et ses pratiques. Le but de l'étude est de mieux comprendre l'apport de la réflexivité dans le cheminement des étudiants de $2^{\mathrm{e}}$ et $3^{\mathrm{e}}$ cycles. Les données proviennent de textes autoréflexifs composés par six étudiantes aux cycles supérieurs, en l'occurrence les auteures de cet article. Ces données ont fait l'objet d'une analyse inductive générale. Trois dimensions identitaires en ressortent: la personne humaine, l'étudiante et l'apprentiechercheure. À la lumière des résultats, la réflexivité comme exercice pédagogique et outil d'accompagnement des étudiants aux cycles supérieurs s'avère bénéfique.

The results presented in this paper stem from an exploratory qualitative co-constructed autoethnography on reflexivity in graduate students. Reflexivity promotes analytical and critical selfassessment of a person's position, role and professional practices. The purpose of this study is to get a better understanding of the contribution of reflective practice in masters' or doctoral students' development. The data is comprised of self-reflective texts written by six graduate students, namely the authors of this article. A general inductive analysis of the data has highlighted three dimensions related to identity: the person, the student and the novice researcher. The results of the study demonstrate that reflexivity is indeed a beneficial pedagogical exercise and mentoring tool for graduate students.

\section{INDEX}

Mots-clés : accompagnement des étudiants, apprentis-chercheurs, construction identitaire, cycles supérieurs, réflexivité

\section{AUTEURS}

\section{ANDRÉANNE GÉLINAS PROULX}

Faculté d'éducation, Université d'Ottawa

ageli079@uottawa.ca

\section{ANNE-SOPHIE RUEST-PAQUETTE}

Faculté d'éducation et École de service social, Université d'Ottawa et Université Laval arues012@uottawa.ca

\section{LILIA A. SIMÕES FORTE}

Faculté d'éducation, Université d'Ottawa

lfort089@uottawa.ca

\section{MEGAN COTNAM-KAPPEL}

Faculté d'éducation,

Université d'Ottawa et Université de Corse Pascal Paoli mcotn025@uottawa.ca 


\section{CAROLINE FALLU}

Department of Foreign Languages,

Kennesaw State University

caroline.fallu@gmail.com

\section{LUCIE BARTOSOVA}

Faculté des sciences de l'éducation, Université de Montréal

luciebartos@gmail.com 